\title{
Research on Yield and Quality Prediction Model of Poplar Based on Modern Information Technologies
}

\author{
Xuning Liu, Aijun Zang, Yuan Li, Shuomei Wu, Wei Shi \\ Department of Computer, Shijiazhuang University, Shijiazhuang, Hebei,China \\ sjzhei@163.com
}

Keywords: poplar, yield, quality, information technology, model

\begin{abstract}
The poplar is important part of shelterbelt trees, so the farmland shelterbelts based on poplar play an important part in the plain, in recent years the research on structure and efficiency mode of farmland shelterbelt are more, while the prediction models of the yield and quality of the polar are much less. The growth model is main material base of poplar growth, the development of information technology provides new methods for the quantitative study of poplar growth model and evaluation. The information technology is used to extract growth character parameters of poplar, and the neural networks is used to establish the relational model of the character parameters and wood materials and wood production of poplar, these models are used to monitor and analyze growth situation and materials of poplar and potential utilization value, so can correctly assess the reasonable use of the poplar, meanwhile provide the basis for the oriented cultivation of poplar management, which have important theory value and practical significance.
\end{abstract}

\section{Introduction}

Poplar is one of the fast-growing reforestation species, and the advantages are that the speed of growth is fast, the yield is high and update speed is easy, which plays an important role in improving the ecological environment and solving the timber problem. Facing with such rich poplar resources, how to cultivate and implement effectively intensive management, and improve forest material, rational development and utilization of timber resources, improve the comprehensive utilization of timber, maximize the value of poplars have become major issues that forest resources cultivation and timber processing and utilization face. This requires timely monitoring, forecasting and analyzing during the course of growth conditions, poplar material and standing potential value[1].

Traditional on-site detection method can only acquire less data, and which are not conducive to study a wide range of poplar parameters, observe a wide range, modern information technologies have the characters of information, dynamic, and provide ideal data source for people to use modern information technologies poplar growth. The main purpose of this research is to explore the feasibility of poplar wood production and quality assessment, and apply modern information technologies to establish the poplars parameters (such as poplar growth width, diameter at breast height, poplar height) and contact of timber yield and quality, which can provide new alternation for poplar wood resource quality and economic value assessment[2].

\section{Research on Remote Sensing Technology}

Remote sensing technology refers that the detecting instruments are used to record the electromagnetic characters of the target from far distance, then explain the characters of the target itself, the nature of variation through analysis and interpretation while it does not contact with goals. Remote sensing system generally includes the following: the information characters of the measured target, information access, transmission of information and records, information processing and information application. In order to extract information from high resolution remote sensing images, then high resolution remote sensing image requires preprocessing. A narrow image pre-processing mainly performs image processing, in order to improve the visual effects of images and lay the foundation for automatic identification, or the image compression is used to reduce the required storage space or transmission time[3]. 
The image pre-processing includes geometric precision correction, data fusion and the shadow removal. The combination of remote sensing information and growth models can be divided into two kinds: the driving method and the initialization parameter method. In this study parametric method is applied, the analog values are consistent with the observed values by adjusting the model parameters, then determine the parameters of the model, when the prediction and yield value of poplar growth are compared with actual observations, and the model genetic parameters increase much higher or lower than the initial values, then a lot of repeatedly debugging are made, making that the parameters model simulation is close to the observed values, the above is an iterative process, and the accuracy is much higher, the drawback is that the process is relatively complex. To sum up, the remote sensing technology has the following characters[4]:

- The detection range is wide and data acquisition is fast. Remote sensing achieves the observation of a large area in a short period of time, and can obtain valuable remote sensing data.

- The observations are repeatedly, and timeliness is good. Remote sensing can be periodically repeated to observe in the same area, which can help to carry out dynamic tracking of changing of many things on earth.

- The information is large. Remote sensing can not only obtain the information of the surface features in the visible band of electromagnetic waves, but also obtain ultraviolet, infrared, microwave and other band information.

- Data collection is convenient, the satellite detection is not constrained by national borders and topographical constraints, and is very beneficial for distribution in the mountains, partial ridge, difficult passage of the forest or woodland exploration.

- The cost is low, compared with traditional methods, the remote sensing technology can greatly save manpower, material and financial resources and time, making high economic and social benefits. After decades of development is made, remote sensing platforms, sensors, or remote sensing information processing, remote sensing applications all have gained rapid development.

\section{Neural Expert System}

Concept of Neural Expert System. As the smart technology, expert system and neural networks have a common goal that they are trying to mimic human intelligence, ultimately create intelligent machines, but the two ways of achieving the objectives of different expert systems depends on logical reasoning and decision poplars, it depends on simulation of human reasoning, but neural networks depends on parallel data processing, it is mainly simulate the ability of human brain to learn the typical expert system rules or the framework is to express knowledge through observation or access to human experts by the rules or framework. Once these rules are stored in the knowledge base, expert system can not change it, and only human manually add, delete, and modify to change knowledge base; For neural network, knowledge is stored as between neurons synapse weights when the training set of input data to the network, these knowledge are obtained in the framework of the expert system structure learning stage, it can learn without human intervention, which means it can learn. However, in a typical expert system, knowledge can be decomposed into a single rule, the user can see and understand the system uses only the block, however the neural network can not regard a single synaptic weight as discrete knowledge, knowledge is embedded into the entire network and can not be broken down into a single block, it actually can not see inside the black box, so the typical expert system and neural network advantages of the hybrid system are called neural expert system for the user, the learning of ability, inductive capacity, stability and parallel processing capacity are much stronger than single artificial neural network reasoning[5].

Composition of Neural Expert System. The poplar growth is a dynamic, random process, and is affected by multi-factors, making the poplars growth and changes in age show complex non-linear. various theoretical models with modeling conditions, applicable conditions and the scope of application, even if these preconditions are satisfied, a variety of conventional models are also difficult to accurately describe the non-status and age of poplar growth linear relationship, modeling 
and prediction results are difficult to achieve the desired results; and the combination of expert system and neural network technology is suitable for the nonlinear problem of modeling and has good fitting ideally for forestry issues.[6]

The system is mainly divided into two parts, expert knowledge and data conversion, user input and output interfaces and knowledge conversion, the inference engine and artificial neural network and knowledge base, expertise and data conversion module are the expert knowledge input module, and knowledge is converted into the knowledge of the rules, and this part of the expert system knowledge constitutes the main source of knowledge about the user input and output interface and knowledge module is responsible for interaction with the user, the symptoms of user input data are regarded as the input of the inference engine reasoning, while the results are returned through the man-machine interface to the user, the inference engine and artificial neural network and knowledge base are the core part of the expert system, including the basic reasoning[7-8];

First expert system is used for the initialization of the expert knowledge rules, after a lot of practice and the results of scientific analysis and appraisal by disease diagnosis expert are regarded as the main source of knowledge of the expert system, manually enter the expert system rules based on the input of expert knowledge to form a knowledge of the rules and are kept into the knowledge base of expert knowledge rules for sample processing, the formation of artificial neural network learning samples, sample are input to the artificial neural network and made training, the system can use while expert system initialization is complete;

Secondly, when the user enters characters of poplar through page, the information of the input data conversion is made, change the data that inference engine can quickly process, the inference engine will first query the knowledge base that already exists in expert knowledge, the expert knowledge base are matched the results. The rules may be given directly to the results, if the results do not match, then the artificial neural network reasoning is used to reason, and reliable results can be got and the results will return to the user, and the corresponding control measures are provided.

\section{Application of BP Network Model}

Determination of BP Network Structure Model. Structure of BP network includes input layer, hidden layer and output layer. Hidden layer has no direct contact with the outside world, through its state the changes affect the relationship between the input and output. The neurons in the same layers have the input connection, and the neurons in the input layer have the input for each neurons in the hidden layer and output layer. We selected five parameters such as poplar growth width, diameter at breast height, poplar height, poplar age and poplar size, these parameters are closed with the growth of poplar and regarded as the input sample data, then the production and quality of poplar are regarded as sample data. A three-layer BP network is used to build the relational model of growth parameters and production and quality model, the number of input layer is 5 , and the number of hidden layer is determined according to the test three nodes; the number of output layer is 2 , so the training pattern includes two modes: normal mode and fault mode, the desired output value of normal mode is 0 , the desired output value of the fault mode is 1 .

Prediction Results. The samples are selected in the collected data for the training of BP neural network, and the trained BP network is used to predict the poplar wood material and production, BP neural network can overcome the shortcomings of manual interpretation and pattern recognition method, which has high precision and is an effective method of prediction. The choice of network structure is regarded as the standard BP network model, the layer of hidden layers is 3 through debugging, and the number of the sample set are considered, change the number of nodes in the training samples and make its accuracy from small to big, when the increase in errors of the number of nodes further is reduced, the value of the threshold is used. Finally, the node number of selected network hidden layer is 9 , the network can quickly converge to the required precision, and the number of input and output number of neurons are 1 and 2 respectively. The samples of training, the input and output data are the following: The one part of sample data of poplar growth are regarded as the training set, while another part of sample data of poplar growth are regarded as the test set for 
the network, the training set samples are used for training, network convergence is in a given standard, the weight value is acquired, the test sample sets prediction and the experimental results are as following Table1:

Table1 Prediction results of improved methods and traditional methods

\begin{tabular}{lccccc}
\hline Improved method & error & mean & Traditional method & error & mean \\
0.571 & 0.006 & 0.575 & 0.679 & 0.007 & 0.675 \\
\hline 0.865 & 0.005 & 0.896 & 0.879 & 0.008 & 0.923 \\
0.812 & 0.006 & 0.834 & 0.877 & 0.016 & 0.926 \\
0.454 & 0.003 & 0.444 & 0.546 & 0.034 & 0.534 \\
0.553 & 0.004 & 0.525 & 0.567 & 0.005 & 0.644 \\
0.357 & 0.012 & 0.387 & 0.493 & 0.415 & 0.455 \\
0.782 & 0.002 & 0.734 & 0.879 & 0.212 & 0.818 \\
0.691 & 0.007 & 0.651 & 0.786 & 0.008 & 0.734 \\
0.771 & 0.001 & 0.742 & 0.890 & 0.002 & 0.768
\end{tabular}

\section{Results and Analysis}

- The choice of training samples, the choice of the training samples should have typical samples, so the sample selection and the number of samples have decisive impact on network performance of the training conditions.

- The network structure, parameter initialization and training termination condition will exist on the network performance of the final training of certain influence because it is to explore the feasibility research of neural network prediction, the network structure and parameters of the beginning has not been further optimized.

\section{Conclusion}

The growth parameters of poplar are an important part of the poplars, and are used to monitor the growing conditions of poplar, or even determine the poplar material; Modern information technology have the characters that observing wide is great, and the quantity of information is influence, the observations are repeated and so on, provides ideal data source for the growth of poplar. The main purpose of this research is to explore the feasibility of modern information technology in production and quality assessment of poplar, so provide new methods for the prediction and assessment of the economic value of poplar.

In this paper modern information technologies are used to extract information of poplar growth, the relational growth model of poplar growth information and poplar growth characters is built. Using these models, we can analyze and assess the growth status of poplar materials and their potential processing and utilization of the value, and provide guidance for the managers, as well as provide the base for poplar management and oriented cultivation. The research makes the combination of modern information technology and growth model and proposes a new way, the methods has important theoretical and practical significance.

\section{References}

[1] Drone K \& Rudemo M. Stem number estimation by kernel smoothing of aerial photos . Canadian Journal of Forest Research.1997,26:1228-1236.

[2] Uuttera J, Haara A, Tokola Iand Maltamo M. Determination of the spatial distribution of poplars from digital aerial photographs, Forest Ecology and Management, 1998, 110 : 275-282.

[3] Hyyppi J, Lnkinen M. Detecting and estimating attributes for single poplars using laser scanner. Photogram. J. Finland.1999,16:27-42.

[4] Gemmel E An investigation of terrain effects Oil the inversion of a forest reflectance model. Remote Sensing of Environment, 1998, 65(2): 155-169. 
[5] Culvenor D S. TIDA: An algorithm for the delineation of poplar clowns in high spatial resolution remotely sensed imagery. Computers \& Geo science. 2002. 28: 33-44.

[6] Eberhart R C ,Dobbins R W. Neural network Pc tools[M] .San Diego : Academic Press ,1990. $280-311$.

[7] Kakeya H ,Dkabe Y. Fast combinatorial optimization with parallel digital computers . IEEE TRANSACTIONS on Neural Networks ,2000,11 (6):1323 - 1331.

[8] Song Xiao Q J, Soh Y C. Robust bock propagation training algorithm for multilayered neural tracking controller.IEEE TRANSACTIONS on Neural Networks ,1999,10 (5):1133 - 1141. 